Note: This is a draft of a paper submitted for publication. Contents of this paper should not be quoted or referred to without permission of the author(s).

To be submitted to the Materials Research Society Fall Meeting, Boston, MA, November 29-December 3, 1999.

\title{
Study of Chromiun-Doped Diamond-Like Carbon by Z-Contrast Imaging and Electron Energy Loss Spectroscopy
}

\author{
X. Fan and S. J. Pennycook \\ Solid State Division \\ Oak Ridge National Laboratory \\ P.O. Box 2008 \\ Oak Ridge, TN 37831 \\ E. C. Dickey \\ Department of Chemical and Materials Engineering, University of Kentucky, \\ Lexington, KY 40506

\footnotetext{
The submitted manuscript has been authored by a contractor of the U.S. Government unde contract No. DE-ACO5-960R22464

Accordingly, the U.S Government retains a

Accordingly, royalty-free license to publish or

reproduce the published form of this

contribution, or allow others to do so, for U.S.

Government purposes."
} \\ prepared by \\ SOLID STATE DIVISION \\ OAK RIDGE NATIONAL LABORATORY \\ Managed by \\ LOCKHEED MARTIN ENERGY RESEARCH CORP. \\ under \\ Contract No. DE-AC05-96OR22464 \\ with the \\ U.S. DEPARTMENT OF ENERGY \\ Oak Ridge, Tennessee
}




\section{STUDY OF CHROMIUM-DOPED DIAMOND-LIKE CARBON BY Z-CONTRAST IMAGING AND ELECTRON ENERGY LOSS SPECTROSCOPY}

X. Fan* ${ }^{\dagger}$ E. C. Dickey*. and S. J. Pennycook ${ }^{\dagger}$

* Department of Chemical and Materials Engineering, University of Kentucky, Lexington, Kentucky, KY, 40506

† Solid State Division, Oak Ridge National Laboratory, Oak Ridge, TN, 37831

\section{ABSTRACT}

Metal-doped diamond-like carbon films were produced for the purpose of an electrochemical nano-electrode. In this study we use Z-contrast scanning transmission electron microscopy to directly observe metal cluster formation and distributions within the chromium-doped carbon films. At low doping ( $-6 \mathrm{at} \% \mathrm{Cr}), \mathrm{Cr}$ is uniformly distributed; at high doping $(\sim 12 \mathrm{at} \% \mathrm{Cr}), \mathrm{Cr}$-rich clusters are formed. Using electron energy loss spectroscopy, we find that the $\mathrm{Cr}$ clusters tend to be metallic-like at low doping levels and carbide-like at high doping levels according to the $\mathrm{Cr} \mathrm{L}_{2,3}$ white line ratios. The carbon is more diamond-like at low doping and more graphite/carbide like at high doping according to the $\mathrm{sp}^{2} / \mathrm{sp}^{3}$ electron percentage measurements.

\section{INTRODUCTION}

Diamond-like carbon (DLC) is a very promising material with wide variety of potential applications due to its unique diamond-like properties[1]. Doping with metals could create a two dimensional array of metal clusters within the diamond-like carbon matrix. Such composite structures are of interest for use as nano-electrodes in electrochemistry[2]. The formation and distribution of the metal clusters within the carbon film are fundamentally important for the physical properties of the material. Here we study the structure and chemistry of chromium-doped DLCs using Z-contrast electron imaging and electron energy loss spectroscopy (EELS). We assess the structure of the diamond matrix by quantifying the percentage of $\mathrm{sp}^{3}$ bonding from the EELS C K-edge, and we investigate the distribution and bonding of Cr within the DLC matrix.

In this work we employ the Z-contrast electron imaging method because it is more directly interpretable than conventional phase contrast imaging[3-5]. Using an annular dark-field detector in the scanning transmission electron microscope (STEM), only the high angle scattering of electrons are collected, where the total scattering intensity is proportional to the square of the atomic number, $\mathrm{Z}$. The $\mathrm{Z}$-contrast image can directly reveal atomic positions as the brighter spots correspond to the positions of heavier atoms. The EELS data can be collected simultaneously through the hole of the dark-field detector allowing direct correlation between the images and spectra.

\section{EXPERIMENTAL}

Diamond-like carbon films of 0.1-5.0 microns thickness were grown on alumina substrates using a plasma chemical deposition technique described elsewhere [6]. The doping of chromium was performed by co-evaporation of chromium into the reactor volume while growing the carbon film $[7,8]$. The carbon film was prepared for transmission electron microscopy by mechanical dimpling from the substrate side and ion beam milling to electron transparency. Two samples doped at low $(\sim 6 \mathrm{at} \% \mathrm{Cr})$ and high levels $(-12 \mathrm{at} \% \mathrm{Cr})$ were examined by Z-contrast imaging in a VG HB603 STEM and a VG HB501. The EELS data were collected on VG HB501 STEM and Philips CM200 transmission electron microscopes.

Fig.1(a) is a phase contrast image of highly doped sample which is similar to a typical amorphous structure. The $\mathrm{Cr}$ is not readily distinguishable. Fig.1(b) is a dark-field Z-contrast image of the same area. Since $\mathrm{Cr}$ atoms are heavier than $\mathrm{C}$, the brighter areas directly correspond to $\mathrm{Cr}$-rich regions. The average $\mathrm{Cr}$-rich cluster size is estimated to be $2 \mathrm{~nm}$ with a separation of $3.5 \mathrm{~nm}$ between clusters. The low-doped sample does not show any $\mathrm{Cr}$-rich regions, indicating uniform $\mathrm{Cr}$ distribution throughout the DLC matrix (picture not shown). 

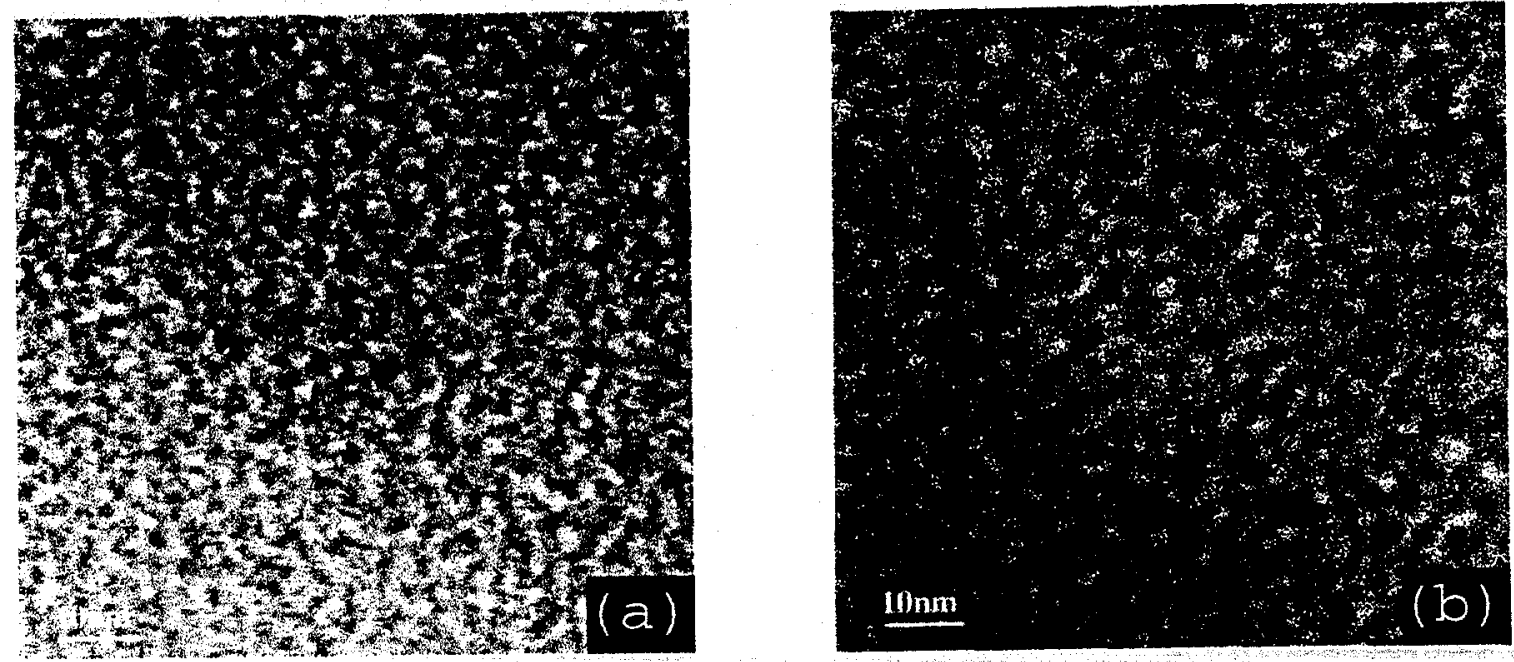

Fig. 1 Chromium doped diamond-like carbon. (a)phase contrast image showing amorphous carbon matrix. (b)Z-contrast image showing $\mathrm{Cr}$ clusters.

The EEL spectra of carbon the K-edge and $\mathrm{Cr}$ L-edge were collected from both samples, as well as from reference samples of chromium carbide $\mathrm{Cr}_{3} \mathrm{C}_{2}$, metallic $\mathrm{Cr}$ and synthetic graphite. Spectra, shown in Fig.2(a-e), were taken in image mode from a scanned area of approximately $100 \mathrm{~nm}^{2}$. Since the highly doped film has uneven $\mathrm{Cr}$ distribution, spatially resolved EELS were also collected on the VG HB 501 with a probe size less than $3 \AA$ from both $\mathrm{Cr}$-rich regions and $\mathrm{Cr}$ deficient regions, as shown in Fig.3. The probe position was determined from the Z-contrast image taken simultaneously with the EEL spectra. Using the intensity ratio of $\mathrm{I}_{\mathrm{C}}$ and $\mathrm{I}_{\mathrm{Cr}}$ and calibrating with the reference sample $\mathrm{Cr}_{3} \mathrm{C}_{2}$, the concentrations of $\mathrm{Cr}$ are found to be an average of 6 at $\%$ for the low doped sample. For the highly doped sample, bright regions show $7-17 \mathrm{at} \% \mathrm{Cr}$ whereas dark regions show $1-3 a t \% \mathrm{Cr}$.

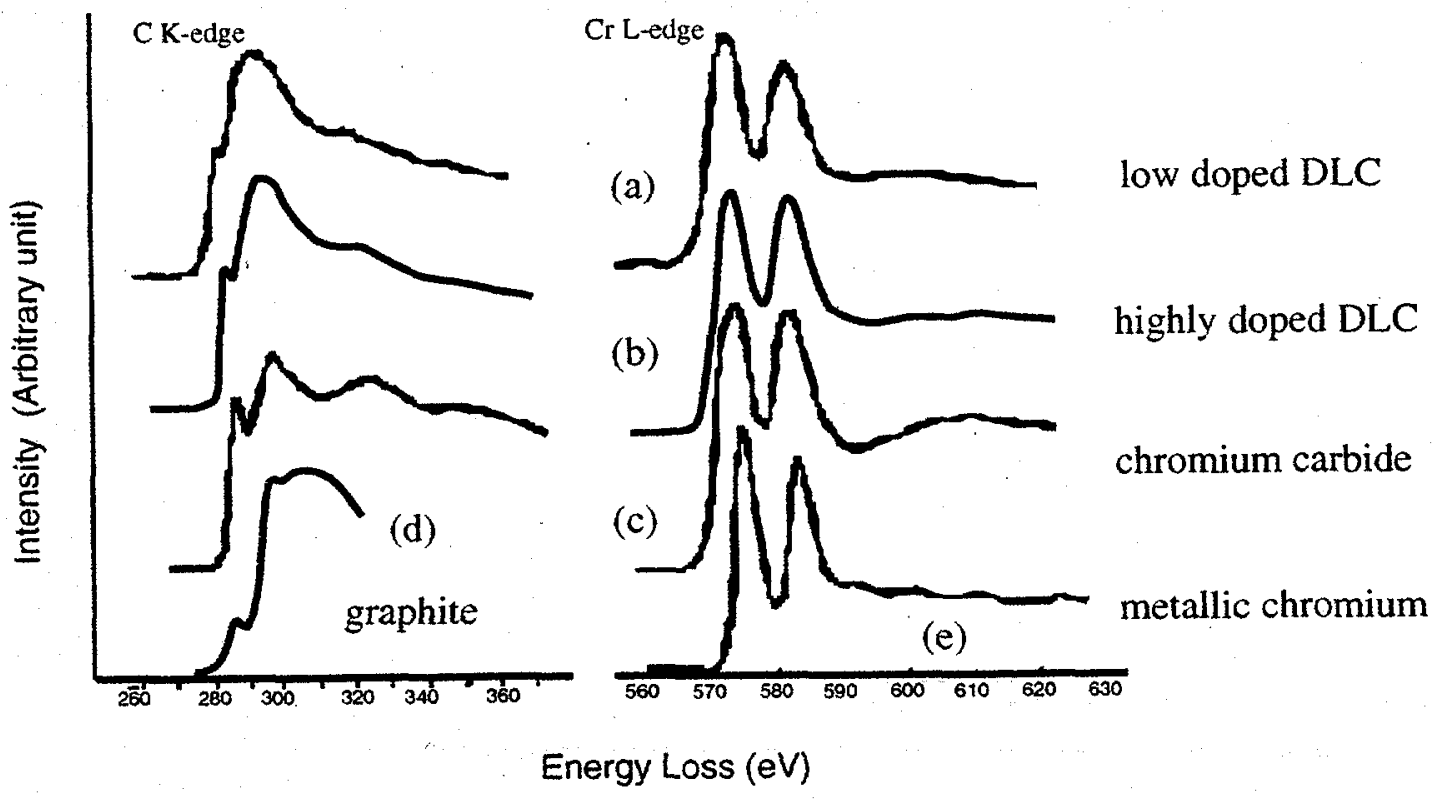

Fig.2 EELS of carbon K-edge(left) and Cr L-edge(right). (a)low doped DLC (b)highly doped DLC (c) Chromium carbide. (d)graphite. (e) metallic $\mathrm{Cr}$. 


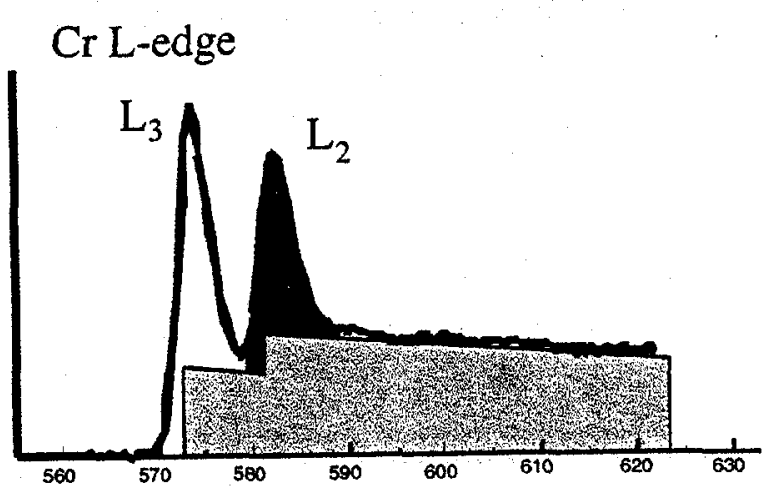

(a)
C K-edge

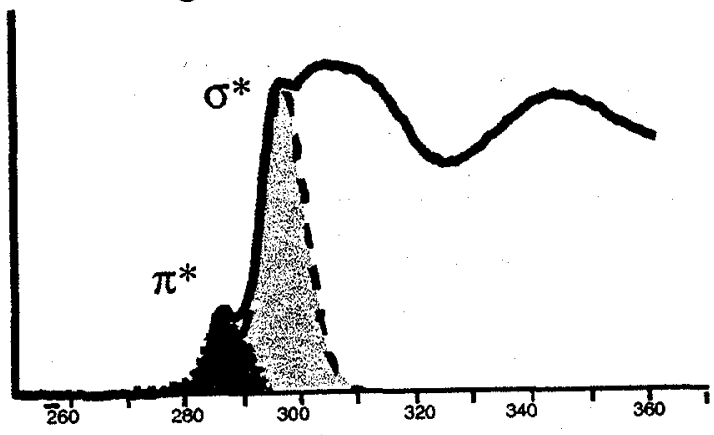

(b)

Fig.4 (a) Using $\mathrm{Cr} \mathrm{L}_{23}$ edge, the whit line ratio $R_{W}$ is defined as the intensity ratio of $\mathrm{L}_{2}$ and $\mathrm{L}_{3}$ edges. The $\mathrm{L}_{2}$ and $\mathrm{L}_{3}$ intensity is obtained by substrate the background.

(b) The carbon $\mathrm{K}$-edge $\sigma^{*}$ peak is fitted with a Gaussian function (light shaded). Subtract it from the original spectrum to get $\pi^{*}$ peak (dark shaded). The total intensity of $I_{\pi+\sigma}$ is integrated under a fixed energy window (280ev-300ev). The $\mathrm{sp}^{2} / \mathrm{sp}^{3}$ ratios is obtained by calibrate $I_{\pi} / I_{\pi+\sigma}$ with pure graphite.

\section{RESULTS}

All chromium $L$-edges have similar features comprising two sharp peaks $L_{2}$ and $L_{3}$, known as "white lines", and a continuum background following the edge. The $\mathrm{L}_{3} / \mathrm{L}_{2}$ white line ratio, $\mathrm{R}_{w}$, is correlated to the electron occupancy and spin pairing in $3 d$-band [9-12]. The variations of the white line ratio are due to the interaction between chromium and surrounding atoms. For example, a large increase of $\mathrm{R}_{w}$ in the chromium alloy $\mathrm{Cr}_{20} \mathrm{Au}_{80}$ reflects the dramatic increase of the magnetic moment [9]. However, it is also found that the white line ratio is sensitive to $\mathrm{Cr}$ cluster size when deposited on a graphite substrate[10].

In our case it is interesting to compare the Cr-doped DLCs with metallic $\mathrm{Cr}$ and chromium carbide. The white line ratios, $\mathrm{R}_{w}$, calculated using the standard method [12], schematically shown in Fig.4(a). The results are listed in Table. I. The white line ratios in low-doped samples are close to metallic $\mathrm{Cr}$. In the highly doped sample, they are close to carbide in the $\mathrm{Cr}$ rich region and metallic in the $\mathrm{Cr}$ deficient region. So, it appears that after a critical doping level, $\mathrm{Cr}$-carbide clusters precipitate from the DLC matrix.

Since the introduction of chromium may change the structure of the carbon, it is also important to evaluate the electronic properties of the carbon. The major difference between a graphite-like carbon and a diamond-like carbon is the absence of $\pi$ electrons in diamond-like material. This feature is shown in the EEL spectra in Fig.2(a-d) where the $\pi^{*}$ peak is at about $284 \mathrm{ev}$. By measuring the intensity ratio of this peak against the following broad $\sigma^{*}$ peak, and then calibrating with the known graphite structure, the percentage of $\mathrm{sp}^{2}$ bonding in the many forms of pure carbon can be determined.

Several methods have been used to measure the $\mathrm{sp}^{2}$ percentage fromt he EELS $\mathrm{C}$ K-edge which involve selection of a fixed window for the $\pi^{*}$ peak integrated intensity $[13,14]$. We find these methods to be insufficient for our study because they do not take into account potential contributions to $\pi^{*}$ integrated intensity from the overlapping $\sigma^{*}$ peak. As can be seen, some of our spectra have higher intensity in the $\pi^{*}$ peak region than that of graphite. This leads to the unrealistic result of higher $\mathrm{sp}^{2} / \mathrm{sp}^{3}$ ratio than graphite. It is believed that the broadening of the $\sigma^{*}$ peak causes 
the increased intensity at $\pi^{*}$ peak region. It was therefore necessary to use a modified version that takes into account this broadening effect [15]. We demonstrate this method for the case of graphite in Fig.4(b). The front edge of the $\sigma^{*}$ peak is partially fitted with a Gaussian function as indicated by the dashed curve. The $\pi^{*}$ function is obtained by subtracting the $\sigma^{*}$ function in the $\pi^{*}$ region, resulting as the shaded small peak. Then the relative peak ratio $I_{\pi} I_{\pi+\sigma}$ is defined as the total intensity of the $\pi^{*}$ peak divided by the total intensity of a fixed energy window in the original spectrum. We choose this range from 280 to $300 \mathrm{ev}$ consistently for all the spectra. For pure carbon materials, the actual $\mathrm{sp}^{2} / \mathrm{sp}^{3}$ ratio can be determined by calibrating with pure graphite for which the percentage of $\mathrm{sp}^{2}$ is $100 \%$.

Our measurements on DLC samples are summarized in Table 1. The low-doped sample shows about $56 \% \mathrm{sp}^{2}$, i.e. $44 \% \mathrm{sp}^{3}$ bonding. In the highly doped material we have the additional complication of carbide formation. The $\mathrm{Cr}_{3} \mathrm{C}_{2}$ standard shows a $\mathrm{C}-\mathrm{K}$ edge structure with a strong peak at about $284 \mathrm{eV}$, the same energy as the $\pi^{*}$ electron peak in graphite (see Fig. 2c). Therefore, the $\mathrm{sp}^{2} / \mathrm{sp}^{3}$ ratio cannot be determined in $\mathrm{Cr}$-rich regions where the $\mathrm{Cr}$ edge indicated carbide formation. The $\mathrm{Cr}$-deficient region shows no evidence of carbide formation, the $\mathrm{Cr}$ edge indicating metallic bonding. Here the $\mathrm{sp}^{2} / \mathrm{sp}^{3}$ ratio could be analyzed, and the percentage of $\mathrm{sp}^{2}$ bonded carbon is found to be almost $100 \%$. We do not believe beam broadening to be a significant factor limiting our spatial resolution; we estimate beam broadening in a $20 \mathrm{~nm}$ thick film to be only $\sim 2 \mathrm{~nm}$, which is comparable to the size of the $\mathrm{Cr}$-rich and $\mathrm{Cr}$-deficient regions. However, if the $\mathrm{Cr}$ signal from the $\mathrm{Cr}$-deficient region was actually due to beam broadening into the adjacent $\mathrm{Cr}$-rich area, the white line ratios would be identical in the two areas. In fact, the white line ratio indicates a change in bonding type, implying we do have the necessary spatial resolution.

Table I. The $\mathrm{Cr}$ concentration at $\% \mathrm{Cr}$, peak intensity ratio $\mathrm{I}_{\pi} / \mathrm{I}_{\pi+\sigma}, \mathrm{sp}^{2}$ percentage and $\mathrm{Cr}$ white line ratio $R_{w}$, obtained from low doped (DLC-1), highly doped (DLC-h) at $\mathrm{Cr}$ rich area (A) and $\mathrm{Cr}$ deficient area $(B)$, the reference samples chromium carbide $\left(\mathrm{Cr}_{3} \mathrm{C}_{2}\right)$, metallic $\mathrm{Cr}$ and graphite.

\begin{tabular}{ccccc}
\hline \hline & $\mathrm{at} \% \mathrm{Cr}$ & $\mathrm{R}_{\mathrm{w}}$ & $\mathrm{I}_{\pi} \pi_{\pi+\sigma}$ & $\% \mathrm{sp}^{2}$ \\
\hline $\mathrm{DLC}-1$ & $6 \%$ & 1.7 & 0.047 & $56 \%$ \\
$\mathrm{DLC}-\mathrm{h}(\mathrm{A})$ & $7-17 \%$ & 1.4 & 0.13 & - \\
$\mathrm{DLC}-\mathrm{h}(\mathrm{B})$ & $1-3 \%$ & 1.7 & 0.11 & $100 \%$ \\
$\mathrm{Cr}{ }_{3} \mathrm{C}_{2}$ & $60 \%$ & 1.35 & 0.15 & - \\
metallic Cr & $100 \%$ & 1.63 & - & - \\
graphite & $0 \%$ & - & 0.11 & $100 \%$ \\
\hline \hline
\end{tabular}




\section{CONCLUSION}

In summary, we have directly imaged the $\mathrm{Cr}$ distribution in $\mathrm{Cr}$-doped diamond-like carbon films by Z-contrast imaging. There is a critical change in $\mathrm{Cr}$ distribution from low to high level doping. The carbon in a low doped sample maintains its diamond-like features; the $\mathrm{Cr}$ is uniformly distributed throughout the matrix and maintains metallic bonding. This implies no strong interaction between carbon and $\mathrm{Cr}$. High $\mathrm{Cr}$ doping leads to the formation of chromium carbide clusters. The residual $\mathrm{Cr}$ in between the clusters remains metallic-like, however the $\mathrm{C}$ looses its $\mathrm{sp}^{3}$-type bonding. Therefore the diamond-like carbon network breaks down at high $\mathrm{Cr}$ doping.

\section{ACKNOWLEDGEMENT}

This research was sponsored by the NSF-MRSEC Advanced Carbon Materials Center under grant No. DMR-9809686, and by the Division of Materials Sciences, U.S. Department of Energy, under contract DE-AC05-96OR22464 with Lockheed Martin Energy Research Corp.

\section{REFERENCES}

1. J. Robertson, Pure \& Appl. Chem. Vol. 66, No. 9, 1789-1796, (1994)

2. R. M. Penner, M. J. Heben, T. L. Longin and N. S. Lewis, Science, Vol.250, 1118$1121,(1990)$

3. S. J. Pennycook and D. E. Jesson, Phys. Rev. Lett. 64, 938-941(1990)

4. S. J. Pennycook, and D. E. Jesson, Ultramicroscopy 37, 14-38 (1991)

5. S. J. Pennycook, "STEM: Z-contrast", in Handbook of Microscopy, ed. by S. Amelinckx, S. Amelinckx, D. van Dyck, J. van Landuyt, G. van Tendeloo, VCH Publishers, Weinheim, Germany (1997) 595-620.

6. V.F.Dorfman and B.N.Pypkin, Surface and Coatings Technology 48, 193 (1991)

7. M. Sunkara, E. Yeap, S.V. Babu, V.G. Ralchenko, B.N. Pypkin, and B.L. Shubekin, "Electrochemical Behavior of Metal-Doped Diamondlike Carbon (Me-DLC) Films", in Electrochemical Society Proceedings Volume 97-32: Diamond Materials V., J.L. Davidson, W.D. Brown, A. Gicquel, B.V. Spitsyn, and J.C. Angus, eds., Published by the Electrochemical Society, pp. 297 - 308, Pennington, NJ, 1997.

8. P. Koduri, M. Sunkara, E.C. Dickey, C. Frasier and S.V. Babu, "Structural and Electrochemical Characterization of Metal-Doped Diamondlike (Me-DLC) Films", Proceedings of 12th Intl. Symp. On Surface Modification Technologies, edited by T.S. Sudarshan, K.A. Khor, and M. Jeandin, Inst. of Materials and American Society of Metals (ASM), pp 357-364, London, U.K., (1998).

9. D. M. Pease, S. D. Bader, M. B. Brodsky, J. I. Budnick, T. I. Morrison and N. J. Zaluzec, Phys. Lett. 114A, No.8,9, 491-494 (1986)

10. L. Lozzi, M. Passacantando, P. Picozzi, S. Santucci and M. De Crescenzi, Z.Phys. D-Atoms, Molecules and Clusters 20, 387-390(1991)

11. T. I. Morrison, M. B. Brodsky and N. J. Zaluzec, Phys. Rev. B32, 3107-3111,(1985)

12. D. H. Pearson, C. C. Ahn and B. Fultz, Phys. Rev. B47, 8471-8478,(1993) 
13. S. D. Berger, D. R. Mckenzie and P.J. Martin, Phil. Mag. Lett. 57, 285-290, (1988) 14. J. Bruley, D. B. Williams, J. J. Cuomo and D. P. Pappas, Journal of Microscopy, 180, Pt 1, 22-32, (1995)

15. B. Rafferty, Chapter 3, Ph.D thesis, Cambridge University, (1997) 